\title{
Pulmonary resection for localized lesions of cryptococcosis (torulosis): a review of eight cases
}

\author{
F. S. S M I T H, P. G I B S O N, T.T. N I CH O L L S, \\ and J.A. S I M P S N \\ Thoracic Surgical Unit, Perth, West Australia
}

\begin{abstract}
Smith, F. S., Gibson, P., Nicholls, T. T., and Simpson, J. A. (1976). Thorax, 31, 121-126. Pulmonary resection for localized lesions of cryptococcosis (torulosis): a review of eight cases. Torulosis is an uncommon, but potentially lethal disease. The aim of this report is to indicate that resection of isolated pulmonary lesions due to torulosis is a safe procedure. Resection has proved useful in the definitive diagnosis and treatment of eight cases seen in this thoracic surgical unit.
\end{abstract}

Cryptococcosis (torulosis) is most commonly due, in man, to infection by Cryptococcus neoformans. This saprophytic, budding, yeast-like, encapsulated micro-organism is widely distributed in soil throughout the world. The organism enters the body through the respiratory tract.

On the basis of postmortem studies, it has recently become apparent that the pathogenesis of primary cryptococcal infection in the lung may often be similar to that of primary tuberculosis (Salyer, Salyer, and Baker, 1974).

The combination of a subpleural nodule and related hilar node involvement may thus be referred to as a primary complex. Its outcome may be:

Benign and self-limiting:

With resolution of the primary complex.

Locally persistent:

Should the pulmonary focus be contained

locally, the parenchymal lesion in the lung will persist and may be recognized radiologically.

Locally progressive:

Continued direct invasion by the organism leads to progressive encroachment on the surrounding lung substance or the adjacent pleural cavity.

Widely disseminated:

Bloodborne spread may involve distant sites, particularly the central nervous system.

The patient may be symptomless, and physical signs may be absent. Commonly, it is the incidental radiological demonstration of a non-specific peripheral pulmonary shadow which draws attention to the disease.
The condition is notoriously difficult to diagnose clinically-the only sure way being the demonstration of the causative organism in the pulmonary lesion. Its recovery from sputum examination is unlikely in the case of a circumscribed parenchymal lesion. It is not surprising that the diagnosis is often not made until after thoracotomy.

\section{PATIENTS}

Eight histologically confirmed cases of pulmonary cryptococcosis in patients who underwent resection were studied (Table). The clinical course and radiological progress of each patient were reviewed, and the present state of the patient was determined.

\section{ILLUSTRATIVE CASE REPORTS}

CASE 2 A 23-year-old foreman was investigated in July 1964 (with negative results) on account of a discrete dense opacity in the right lower lobe, which had been found on mass miniature radiography (Fig. 1). A review of chest films, taken five years previously while the patient was in the Navy, revealed a soft opacity in the right lower lobe. The patient declined operation for five months while under observation until the mass had enlarged appreciably (Fig. 2). Right lower lobectomy revealed torulosis. The lesion was well encapsulated. The cerebrospinal fluid (CSF) was normal. No chemotherapy was given. The patient remains well nine years after operation. This pulmonary lesion had remained locally persistent and inactive for at least five years before it became locally progressive. 


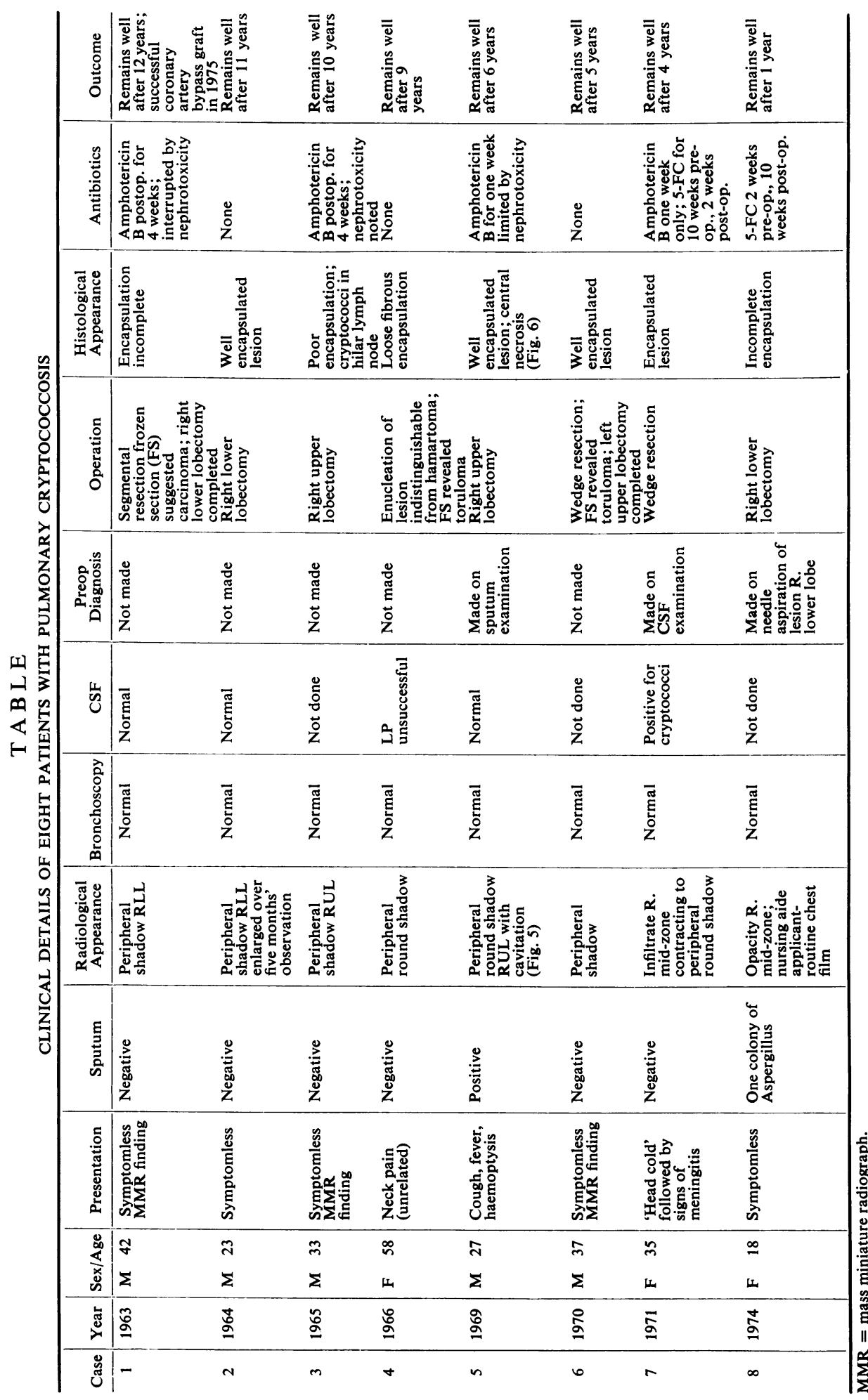

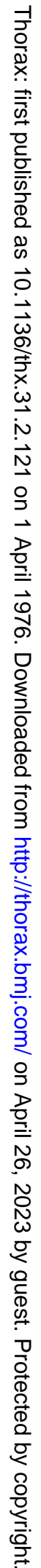




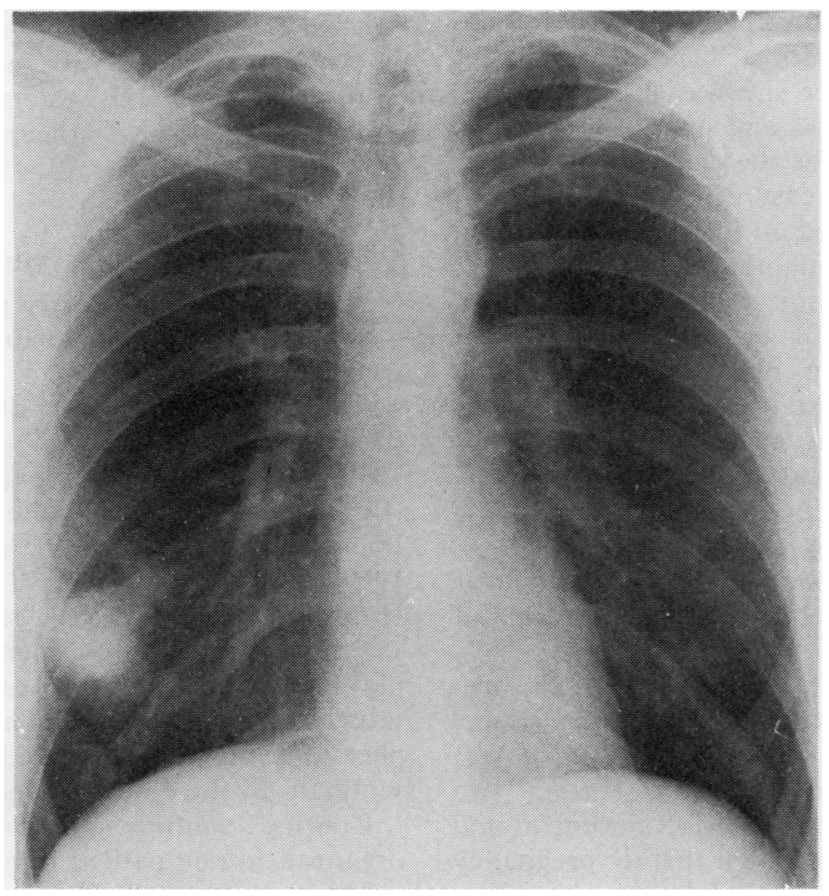

FIG. 1. Case 2. Toruloma right lower lobe.

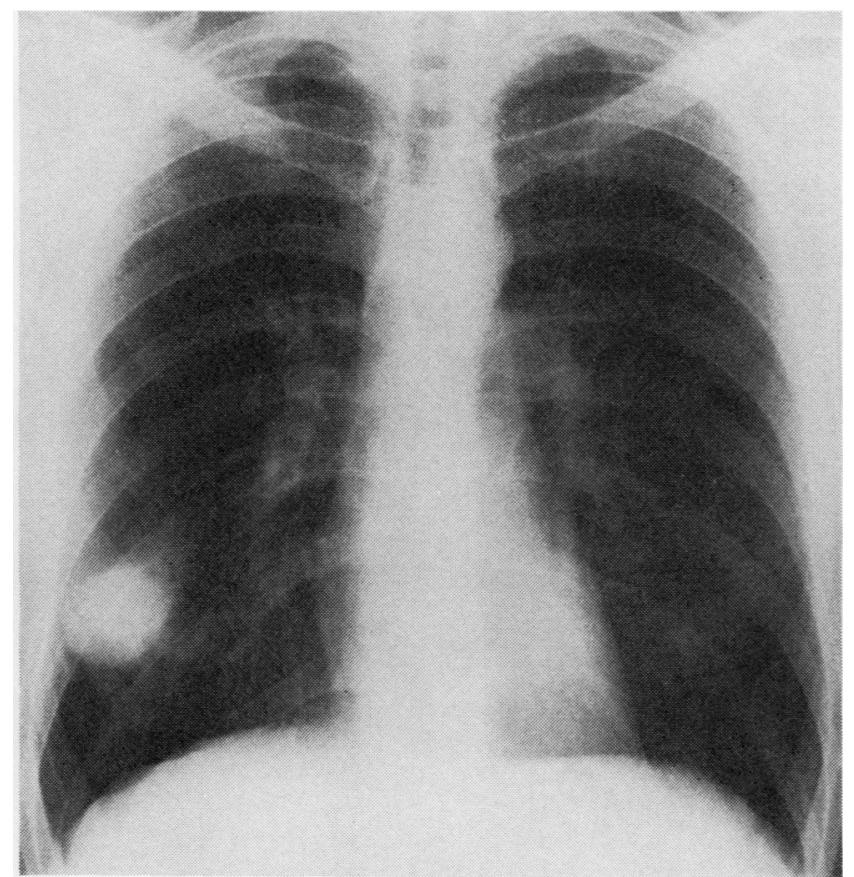

FIG. 2. Case 2. Shows increase in size over five months. 
CASE 7 An aboriginal woman of 35 years was treated with antibiotics for a 'head cold' in early July 1971. A week later she was flown to Perth with drowsiness, headache, and signs of meningitis. Rhonchi were heard over the right chest. A lumbar puncture showed yeast cells in the CSF. A chest film (Fig. 3) showed an infiltrate in the right lung. A course of amphotericin B was begun. Raised intracranial pressure due to basal cisternal blockage was relieved by an Ommaya reservoir and frequent extraction of CSF. 5-Fluorocytosine (5-FC) was administered from 15 July 1971 in a dose of $200 \mathrm{mg} / \mathrm{kg}$ per day and this abolished the cryptococcal meningitis. By 23 July 1971 the lung infiltrate had cleared, except for a rounded opacity in the right midzone (Fig. 4) which then remained virtually unchanged over several weeks, despite continued administration of 5-FC.

On 18 October 1971 wedge resection of a toruloma, $1.5 \mathrm{~cm}$ in diameter in the right upper lobe, was carried out under intravenous 5-FC cover, which was continued orally for a further two weeks. The patient's recovery was uneventful and she remains well. She has had a further pregnancy without incident.
Comment This patient presented with evidence $\overrightarrow{\vec{\rho}}$ of both pulmonary involvement and distant spread to the central nervous system. Once the meningitis $\frac{\bar{\sigma}}{\sigma}$ had been controlled, the pulmonary focus was resected uneventfully.

\section{DISCUSSION}

Nearly 40 years ago, early surgical removal of a localized area of pulmonary torulosis was first $\vec{\omega}$ considered (Taber, 1937).

By 1945 , the first report of pulmonary resection $\times$ for this condition had appeared (Dormer et al., $\stackrel{\omega}{\rightarrow}$ 1945). Sporadic reports over the next 25 years i (Froio and Bailey, 1949; Berk and Gerstle, 1952; $\vec{N}$ Poppe, 1954; White and Arany, 1958; Perkins, 1969; Hatcher et al., 1971; Epstein, Cole, and Hunt, 1972) indicated the safety of resection.

Despite the differing operative procedures em- o ployed in this series, the results were uniformly satisfactory, with an absence of surgical complications. In no case did the condition disseminate as the result of operation.

Routine sputum examination revealed the organism in one patient only.

Standard bronchoscopy was unhelpful as a

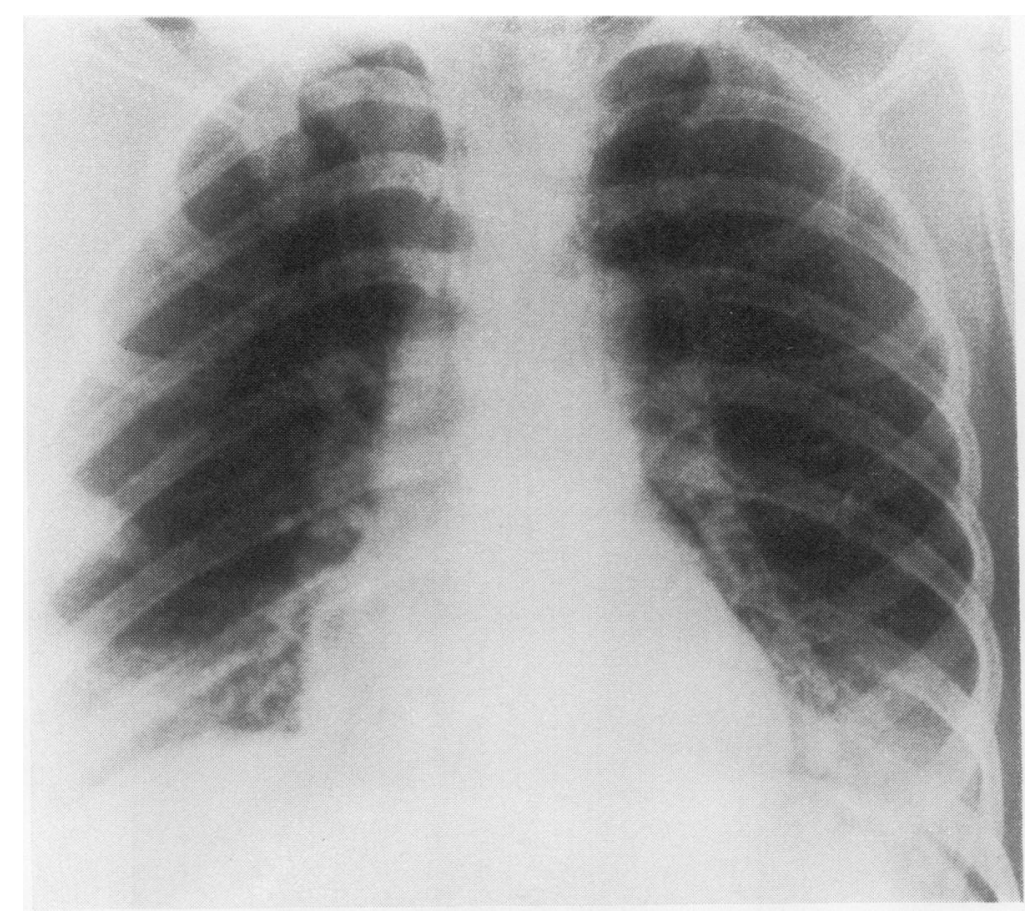

FIG. 3. Case 7. Infiltrate right lower lobe. 


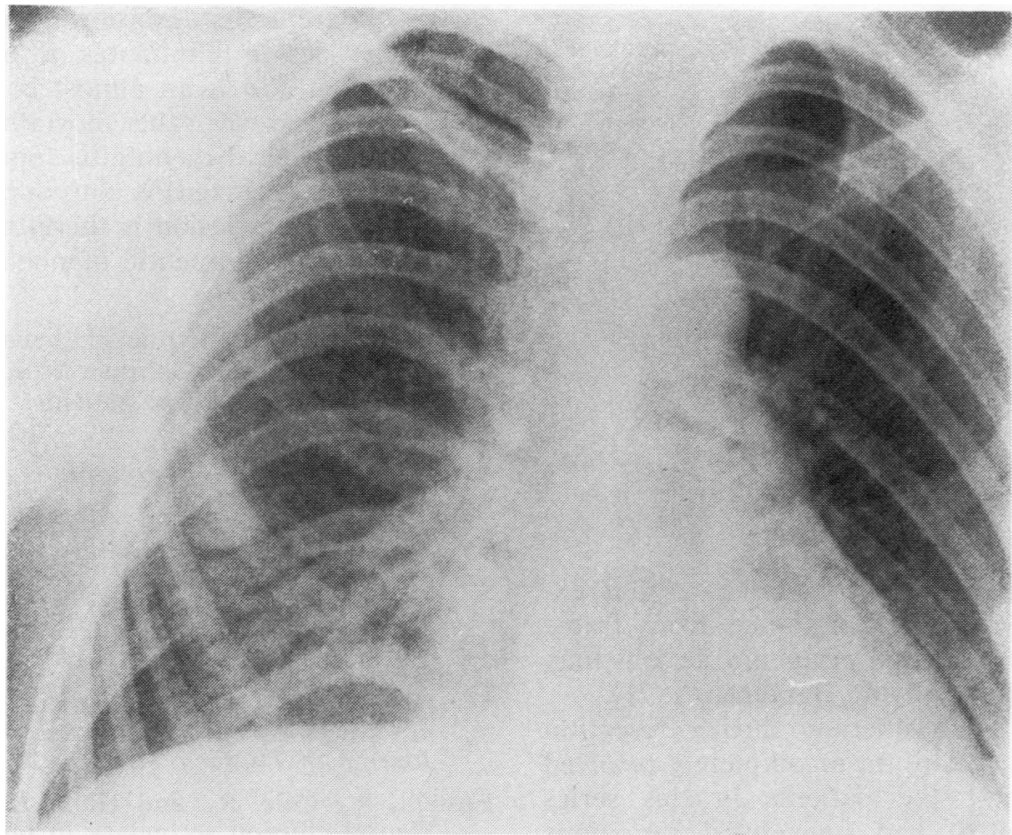

FIG. 4. Case 7. Peripheral round shadow right lower lobe.

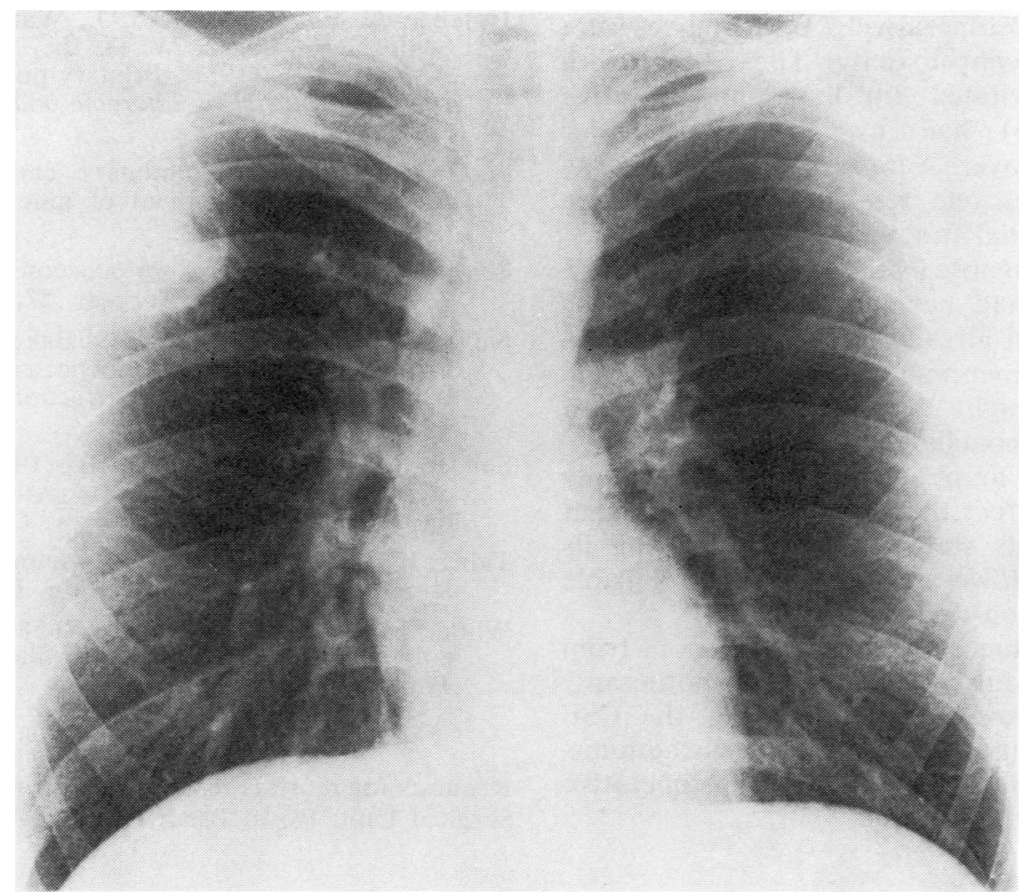

FIG. 5. Case 5. Unusual cavitated lesion in the right upper lobe. 


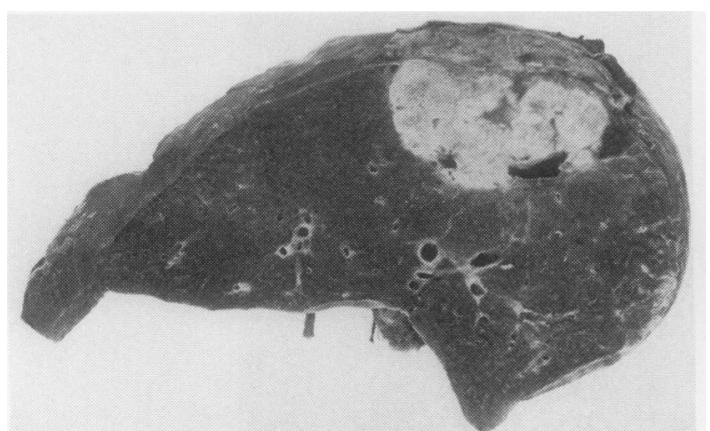

FIG. 6. Case 5. Cut surface of right upper lobe showing lesion.

diagnostic aid. Recent reports indicate that examination of the material obtained from transbronchial brushing of such peripheral lesions may be more rewarding (Soll and Bergeron, 1971).

The place of chemotherapy during resection for this condition is still an incompletely resolved question. Three of the patients in this series (cases 2, 4, and 6) had uneventful resections without the use of chemotherapy. Their lesions were encapsulated.

Two others (cases 1 and 3), with spread beyond the periphery of the main lesion, received amphotericin B postoperatively. Both courses were interrupted by nephrotoxicity. The patient with the unusual cavitated toruloma and positive sputum (case 5) had his lobectomy under amphotericin B cover. Administration of the drug was stopped after one week because of severe impairment of renal function.

The patient diagnosed preoperatively by needle biopsy (case 8 ) had her lobectomy under 5-FC cover. The patient already receiving $5-\mathrm{FC}$ to control cryptococcal meningitis (case 7) had her lesion resected a fortnight before her three-month course had been concluded.

Until recently amphotericin B was the only drug known to affect this organism, but frequent serious side effects were encountered during its administration. However, 5-FC is now available and is relatively non-toxic.

Perkins (1969) successfully resected lesions from five patients without co-incidental chemotherapy. $\mathrm{He}$ advised, however, examination of the CSF to exclude occult meningeal spread. Drug administration may diminish the risk of postoperative spread (Hatcher et al., 1971).
It is apparent that excision of a circumscribed $\overrightarrow{\vec{s}}$ pulmonary lesion eliminates it as a focus for $\overrightarrow{0}$ dissemination and is an almost complication-free $\frac{}{0}$ operation. However, the mortality from estab- क lished cryptococcal meningitis (once spread from $\Phi$ the lungs has occurred) is considerable. Resection of the pulmonary lesion is therefore a reasonable 5 diagnostic and therapeutic manoeuvre.

Our thanks are due to the physicians of the Royal Perth and Sir Charles Gairdner Hospitals for investi- $\overrightarrow{\vec{x}}$ gating and referring these patients.

\section{REFERENCES}

Berk, M. and Gerstle, B. (1952). Torulosis (cryptococcosis) producing solitary pulmonary lesion;음 report of a four-year cure with lobectomy. Journal of the American Medical Association, $\rightarrow$ 149, 1310.

Dormer, B. A., Friedlander, J., Wiles, F. J., and Simson, F. W. (1945). Tumour of the lung due $\vec{\theta}$ to Cryptococcus histolyticus (blastomycosis). Journal of Thoracic Surgery, 14, 322.

Epstein, R., Cole, R., and Hunt, K. K., Jr. (1972). Pleural effusion secondary to pulmonary cryptococcosis. Chest, 61, 296.

Froio, G. F. and Bailey, C. P. (1949). Pulmonary cryptococcosis. Report of a case with surgical cure. Diseases of the Chest, 16, 354.

Hatcher, C. R., Jr., Sehdeva, J., Waters, W. C., III, 을 Schulze, V., Logan, W. D., Jr., Symbas, P., and Abbott, O. A. (1971). Primary pulmonary cryptococcosis. Journal of Thoracic and Cardiovascular Surgery, 61, 39.

Perkins, W. (1969). Pulmonary cryptococcosis: A report on the treatment of nine cases. Diseases of the Chest, 56, 389.

Poppe, J. K. (1954). Cryptococcosis of the lung. Journal of Thoracic Surgery, 27, 608.

Salyer, W. R., Salyer, D. C., and Baker, R. D. (1974). Primary complex of cryptococcus and pulmonary윽 lymph nodes. Journal of Infectious Diseases, 130, 47.

Soll, E. L. and Bergeron, R. B. (1971). Pulmonary cryptococcosis: a case diagnostically confirmedo by transbronchial brush biopsy. Chest, 59, 454.

Taber, K. W. (1937). Torulosis in man. Journal of the N American Medical Association, 108, 1405.

White, M. and Arany, L. S. (1958). Resection in pulmonary cryptococcosis (torulosis). Journal of Thoracic Surgery, 35, 402.

Requests for reprints to: F. S. Smith, FRCS, Thoracic Surgical Unit, Royal Perth Hospital, W. Australia. 